\title{
The impact of Hashimoto's disease on female fertility: a systematic review
}

\section{Antigoni Sarantaki*, Kyriaki Tsiorou, Kleanthi Gourounti}

Department of Midwifery, University of West Attica, Egaleo, Athens, Greece

Received: 02 December 2021

Revised: 05 January 2022

Accepted: 06 January 2022

\section{*Correspondence:}

Dr. Antigoni Sarantaki,

E-mail: esarantaki@uniwa.gr

Copyright: ( ) the author(s), publisher and licensee Medip Academy. This is an open-access article distributed under the terms of the Creative Commons Attribution Non-Commercial License, which permits unrestricted non-commercial use, distribution, and reproduction in any medium, provided the original work is properly cited.

\begin{abstract}
Hashimoto's disease (HT) is the most common autoimmune disease, affecting 5-15\% of women of reproductive age. Recent studies have shown that Hashimoto's thyroiditis can be a risk factor for infertility in women. The aim of the present study is to explore the impact of Hashimoto's disease on female fertility. This systematic review followed the preferred reporting items for systematic reviews and meta-analyses (PRISMA) guidelines. Databases searched included Medline, PubMed, EMBASE and Google Scholar. Search terms included Hashimoto's disease, Hashimoto's thyroiditis, thyroid autoimmunity, autoimmune thyroiditis, women, females, infertility and every possible combination of them. The exclusion criteria of this systematic review involved articles written in a language other than English, other disorders that also affect the female fertility, women with infertile partners, with abnormal karyotype or/and with abnormal body mass index (BMI). The search strategy identified 150 unique items. After the initial screening process, 50 studies underwent full text review and 5 studies were finally included in the systematic review, since they met all the inclusion criteria. Four of the included studies found that Hashimoto's thyroiditis can have a negative effect on female fertility and especially in patients of advanced age. Women with autoimmune thyroiditis might be at risk of ovarian insufficiency because of the decline in ovarian reserve. Consequently, patients with positive anti-thyroid peroxidase (TPO) antibody findings, who wish to start a family, should be aware of their lower reproductive life span. Further randomized controlled trials can clarify the impact of Hashimoto's disease on female fertility.
\end{abstract}

Keywords: Autoimmune thyroiditis, Hashimoto's disease, Hashimoto's thyroiditis, Thyroid autoimmunity, Women, Females, Infertility

\section{INTRODUCTION}

Undiagnosed and untreated thyroid disease can be a cause of infertility as well as sub-fertility. The terms subfertility and infertility are often used interchangeably, but they aren't identical. Subfertility is a delay in conceiving. Infertility is the inability to conceive naturally after one year of unprotected regular sexual intercourse. Both these conditions have important medical, economical, and psychology implications in the society. ${ }^{1}$ Thyroid dysfunction can affect fertility in various ways resulting in anovulatory cycles, luteal phase defect, high prolactin (PRL) levels, and sex hormone imbalances. Therefore, normal thyroid function is necessary for fertility, pregnancy, and to sustain a healthy pregnancy, even in the earliest days after conception.

Hashimoto (HT) is a chronic autoimmune disease in which destruction of the thyroid occurs as a result of lymphocyte infiltration. It is the most common autoimmune disease, affecting $5-15 \%$ of women of reproductive age and 20$30 \%$ of the total population. It is caused by an increased level of titers of antibody against thyroid peroxidase (TPO) and thyroglobulin (TG). As a result, changes are observed in the level and metabolism of thyroid hormones, which lead to unspecified physical and psychological symptoms. 
From the etiological point of view, the most significant are the genetic factors. However, environmental factors may provoke the immune system to attack the thyroid tissue. Some of these environmental factors are the specified stress, toxification, microbiota dysbiosis and under- or over-nutrition, to name just a few. ${ }^{2}$ In cases of Hashimoto's thyroiditis with overt hypothyroidism, replacement therapy with L-thyroxine will be adapted to the thyroid stimulating hormone (TSH) level. ${ }^{3}$

According to World Health Organization (WHO), infertility is a disease of the male or female reproductive system defined by the failure to achieve a pregnancy after 12 months or more of regular unprotected sexual intercourse. Estimates suggest that between 48 million couples and 186 million individuals live with infertility globally. ${ }^{4}$ However, there are no data, explaining the prevalence of infertility in women with autoimmune diseases and specifically in those, that occur during the reproductive age and influence the endocrine system. Autoimmune thyroid dysfunction, is the most common of these diseases. Graves' disease (GD) affects around 1\% of the population, while Hashimoto's thyroiditis (HT) affects around $3 \% .^{5}$ In addition, they consist of hyper- and hypothyroidism, both of which can interfere with the mechanism of ovulation and with sex hormone metabolism, affecting fertility and if gestation takes place, it may have more possibilities for early pregnancy losses and it may affect the fetal health negatively. ${ }^{6}$ The incidence of miscarriage, mainly happens in the first trimester and it was found to be higher (6-15\%) in patients with hypothyroidism in contrast to healthy women $(2.2 \%)$ with a higher risk of miscarriage as a result of the increase in maternal TSH concentrations. Except for thyroid function, TPO $\mathrm{Ab}$ were found to be associated with an increased miscarriage rate up to $43.9 \% .^{7}$ Despite the probability that thyroid dysfunctions and autoimmunity can negatively affect the fertility of women of reproductive age, no specific studies have been carried out to measure the prevalence of infertility in groups of HT. ${ }^{6}$ Similarly, the American college of obstetricians and gynecologists does not advise universal screening in pregnancy for either thyroid function or antithyroid antibodies. ${ }^{8}$

The objective of the present systematic review is to explore the literature, so as to estimate the impact of Hashimoto's disease on female fertility, while focusing on recently performed studies.

\section{METHODS}

The preferred reporting items for systematic reviews and metanalyses (PRISMA) guidelines were followed to conduct a systematic review of the literature. ${ }^{9}$

\section{Information sources}

The search strategy for the review was primarily directed toward finding published papers in journals and conference proceedings via the widely accepted literature search engines and databases EMBASE, MEDLINE and Google Scholar. No language limits were applied and foreign texts were translated. There were date limits since the studies, that were included in the present systematic review, have been written during the last decade. Specifically, the initial results were downloaded in April 2021 with updated searches run in June 2021.

\section{Search strategy}

For the search, the researchers focused on selected keywords, based on the aimed scope of the literature review. Appropriate subject headings and special keywords were used for each database. Those keywords and terms included the following subject headings: Hashimoto's thyroiditis, Hashimoto's disease, thyroid autoimmunity, autoimmune thyroiditis, infertility, sterility, women, females and every possible combination between them. Those keywords and terms were both searched in titles, abstracts and full texts of scientific studies. At the beginning, the two researchers individually extracted information from articles for cross-checking. After reviewing a few articles together, they reached consensus on what to extract from the articles. Then, the researchers split up the work. They maintained frequent communication during the data extraction process. Articles that were hard to decide were discussed between them. In the subsequent phase, all three researchers reviewed the abstracts (and keywords) of the collected papers with respect to the defined set of inclusion and exclusion criteria, as followed. Although the selection process was primarily based on the review of paper abstracts and keywords, in the cases where these two were insufficient, the researchers also considered parts of the introduction, contribution and conclusion sections.

\section{Eligibility criteria}

The present study was limited to articles that were written in English and to those that had the most recent literature. Every recent study that gave information about Hashimoto's disease and female fertility was included in the study selection. As for the characteristics of the study participants, every infertile woman of reproductive age who had only an autoimmune disease and especially with Hashimoto's disease was included in the initial screening process. Additional inclusion criteria included the normal karyotype and the normal body mass index (BMI). Women who had further disorders that also affect the female fertility or women with infertile partners, were both excluded from the study selection. Women who did not have an active sexual life were excluded from the study selection as well. With the term "infertile", were defined women who were unable to achieve pregnancy after 12 months of regular and unprotected sexual intercourse. Articles written in a language other than English, were excluded from this review as well as the studies that did not provide causal information on Hashimoto's disease and infertility. 
Table 1: Exclusion criteria.

\begin{tabular}{|ll|}
\hline S. no. & Exclusion criteria \\
$\mathbf{1}$ & $\begin{array}{l}\text { Articles written in a language other than } \\
\text { English }\end{array}$ \\
\hline $\mathbf{2}$ & $\begin{array}{l}\text { Other disorders that also affect the female } \\
\text { fertility }\end{array}$ \\
\hline $\mathbf{3}$ & Women with infertile partners \\
\hline $\mathbf{4}$ & The abnormal karyotype \\
\hline $\mathbf{5}$ & The abnormal body mass index (BMI) \\
\hline
\end{tabular}

Table 2: Search terms used in the study.

\begin{tabular}{|lll|}
\hline Search terms used in the study & \\
Women/ & $\begin{array}{l}\text { Hashimoto's disease/ } \\
\text { Hashimoto's thyroiditis/ }\end{array}$ & $\begin{array}{l}\text { Infertility/ } \\
\text { females } \\
\text { thyroid autoimmunity/ } \\
\text { autoimmune thyroiditis }\end{array}$ \\
\hline
\end{tabular}

\section{Study extraction and quality appraisal}

The data were extracted by authors independently, using a data extraction format prepared in a Microsoft excel 2010 spreadsheet. The extracted data were the first author's name, publication year, country, design, research tools and prevalence of ovarian dysfunction among Hashimoto disease patients. The quality of each study was assessed using the modified Newcastle-Ottawa scale (NOS) for cross-sectional studies. ${ }^{10}$ Studies were included with a score of 5 and more on the NOS. ${ }^{11}$ Each study's quality was evaluated independently by the three authors, and any disagreements were resolved by discussion and consensus.

\section{RESULTS}

The search strategy identified 150 unique records. After the initial screening process and after having read the titles and the abstracts of those studies, ten studies underwent full text review. Five studies were excluded because they did not meet the full inclusion criteria. At this stage, studies were excluded from the study selection for the following reasons: Some studies were not too recent, as they were written 10 years ago. Also, some women had additional health problems, apart from Hashimoto's disease, that also affected fertility or there were male factors that had a negative impact on fertility as well. Five studies were ultimately found to meet all the inclusion criteria and were ultimately included in the systematic review. The four eligible studies were conducted between 2014 and 2020 with sample sizes ranging from 52 to 165 subjects. These studies were conducted across several countries, including three studies in Europe, one study in Iran and one study in Brazil. The various settings included hospitals/centers like the Centre for Women's Health, Tubingen University Hospital, Department of Endocrinology and Metabolism and Department of Biochemistry, Yildirim Beyazit University Medical School Ataturk Teaching and Research Hospital, Bilkent, Ankara, Turkey, the infertility center of Shiraz University of Medical Sciences, Hazrate Zeinab Hospital and the Endocrinology clinic for thyroid autoimmune diseases, School of Medical Sciences, University of Campinas (UNICAMP), Sao Paulo, Brazil. All studies, aimed to determine the impact of Hashimoto's thyroiditis on female fertility

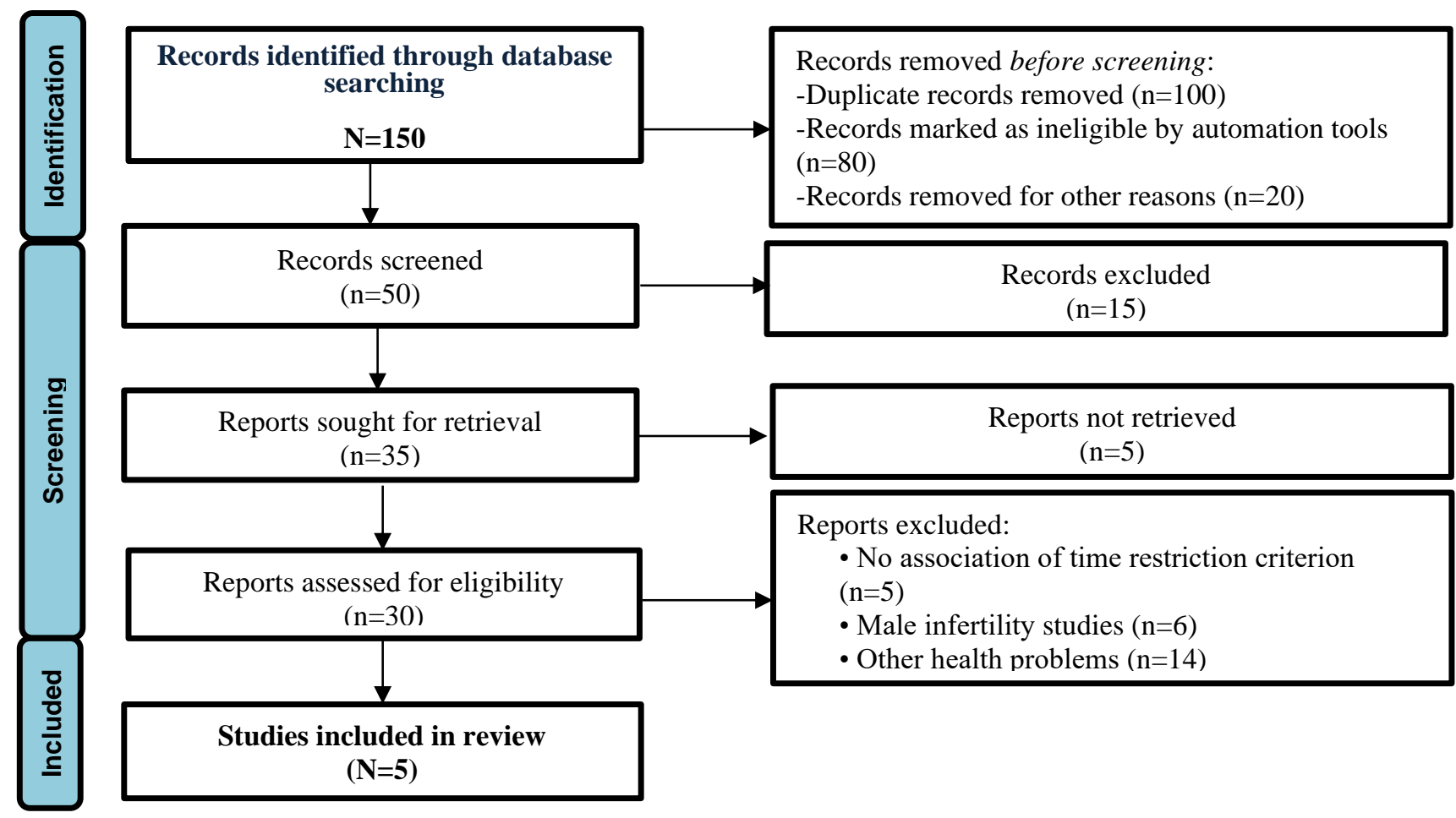

Figure 1: Selection process of included studies. 


\section{Hashimoto's disease and premature ovarian failure}

One study that conducted in Germany included 52 women (aged between 18 and 40 years) with premature ovarian failure, diagnosed at the Centre for Women's Health, Tubingen University Hospital. Diagnostic criteria for premature ovarian failure were amenorrhea, lasting more than 4 months and an Anti-Mullerian hormone (AMH) level below the age-appropriate range. AMH has been shown to be the best serological marker to evaluate ovarian reserve. This hormone was measured by electrochemiluminescence immunoassay (ECLIA; Roche Diagnostics, Mannheim, Germany). The test measures concentrations between 0.07 and 164 pmol/l. Depending on age, normal values range from 5.5 to $67.79 \mathrm{pmol} / \mathrm{l}$. All participants were tested for chronic infections that might have had a negative influence on fertility. Screening included: a standard blood analysis, rheumatoid factor, anti-cyclic citrullinated peptide antibodies, antinuclear antibodies, anti-neutrophil cytoplasmic antibodies, thyroid peroxidase antibodies, thyroglobulin antibodies, TSH receptor antibodies, and antibodies to mitochondria, adrenal cortex cells and islet cells. The reason for presentation in $40.4 \%(n=21)$ of participants was a desire for pregnancy. In 21 participants, at least one autoimmune disease was detected. From those 21 participants, 17 (32.7\%) preceded a diagnosis of Hashimoto's disease. In women with Hashimoto's disease, thyroid peroxidase antibodies were positive in $29.4 \%(n=5)$, thyroglobulin antibodies were positive in $17.6 \% \quad(n=3)$ and both antibodies were positive in $52.9 \%(\mathrm{n}=9)$. Furthermore, clinically euthyroid women (17.3\%) were antibodypositive. The average value for TSH was $1.65 \mathrm{mIU} / 1$, range from 0.01 to $6.21 \mathrm{mIU} / \mathrm{l}){ }^{12}$

\section{Hashimoto's disease and Anti-Mullerian hormone - ovarian reserve}

Another study, conducted in Ankara, Turkey, included 85 women with an autoimmune thyroid disease (AITD) and 80 healthy women, all under 40 years of age. Women with AITD were positive for one or more of the serum thyroid antibodies and the ultrasonography (USG) findings consistent with thyroiditis. Healthy controls were chosen from among the subjects attending outpatient clinics for check-up. Women in the control group had regular menstrual cycles, normal thyroid function, and normal thyroid gland at ultrasonography (USG). An early morning sample of blood was obtained from both groups of women during the follicular phase (days 3-5). This blood was used for the measurement of $\mathrm{AMH}$, inhibin $\mathrm{B}$, gonadotrophins, estradiol, testosterone, $17 \mathrm{OH}$ progesterone, and dehydroepiandrosterone sulfate. Patients had at least one type of thyroid antibody positivity: anti-Tg in $51(60 \%)$, anti-TPO in $64(75.3 \%)$, and both in $30(35 \%)$. Patients and controls had regular menstrual cycles. The serum levels of gonadotrophins, sex steroids, inhibin B, and free thyroid hormones were similar in the two groups whereas TSH was higher in women with AITD. The patients with AITD had significantly lower levels of AMH than controls. Also, compared with controls, the AITD group had a significantly lower number of pregnancies and live births. $^{13}$

Other research, conducted in Iran, included 98 infertile women (age between 18 and 42 years). All women had TSH level higher than $3 \mathrm{mIU} / \mathrm{l}$, normal serum prolactin, BMI $<35 \mathrm{~kg} / \mathrm{m}^{2}$ and they did not have other autoimmune diseases. Those women were divided into two groups based on the positive or negative anti-TPO levels. Data were collected by a form containing demographic data, TSH, thyroxine $\left(\mathrm{T}_{4}\right)$, anti-TPO antibody, prolactin, AMH, FSH, luteinizing hormone (LH), and antral follicular count (AFC). The results presented that the patients' mean age, BMI, duration of infertility, mean prolactin, TSH, thyroxine and FSH level did not differ significantly between those two groups. However, there were significant differences between the two groups on $\mathrm{AFC}$ and $\mathrm{AMH}$, indicating significantly lower mean AFC and AMH levels in the anti-TPO positive group. In women younger than 35 years, only mean AFC level was lower, but in the older age group, both AMH and AFC levels were lower in the anti-TPO positive group. ${ }^{14}$

Another study conducted in Turkey, included 30 adolescent girls newly diagnosed as HT, with presence of high thyroid antibodies with gland heterogeneity in ultrasound and 30 healthy teenager controls. Anti-ovarian antibody (AOAb), LH/FSH ratio, estradiol, AMH, inhibin$\mathrm{B}$, total testosterone, antral follicle count, ovarian volumes and uterine length were estimated in both groups. After that, the clinical, laboratory, and ultrasound data of the HT and control groups were compared. The results showed no important differences between the teenagers with HT and healthy controls in relation to $\mathrm{LH} / \mathrm{FSH}$ ratio, estradiol and inhibin-B levels. Also, AOAb, AMH and total testosterone levels were higher in HT group. ${ }^{15}$

\section{Hashimoto's disease and infertility}

A Brazilian study, conducted in the endocrinology clinic for thyroid autoimmune diseases, included 66 women (age between 18 and 60 years old) with Hashimoto's thyroiditis. None of the women in the study had history of seeking infertility treatment. All participants were interviewed by the same obstetrician-gynecologist, using a structured questionnaire specially made for the research, aiming to collect information about their reproductive history. After the interview, the women's medical charts were analyzed to determine the characteristics of their disease, the date of their diagnosis, the period since the diagnosis, and the results of laboratory and imaging tests confirming the diagnosis of possible associated autoimmune diseases. From a sample of 66 women, 31 had fertility problems. The average age of those women was 44.7 years. The average age at menarche was 12.4 years and most of those women started their sexual life at the age of 18.5. According to the diagnosis, at an average age of 39.2 years, the women were diagnosed with Hashimoto's disease. Ten women had goiter and 15 had a thyroid 
nodule. Twenty-seven women were anti-TRO and anti-Tg positive and 4 women had additional autoimmune diseases. Also, 31 women were found to have family history of thyroid diseases ( 2 women had a family history of Grave's disease, 11 of Hashimoto's disease and 18 of other autoimmune diseases). In a population of 66 women with HT enrolled in that study, the prevalence of infertility was $47.0 \%{ }^{16}$

Table 3: Characteristics of the included studies.

\begin{tabular}{|c|c|c|c|c|c|}
\hline $\begin{array}{l}\text { Study/ } \\
\text { year }\end{array}$ & $\begin{array}{l}\text { Desig } \\
\text { n }\end{array}$ & Country & $\begin{array}{l}\text { Sample } \\
\text { size }\end{array}$ & Tools & Main results \\
\hline $\begin{array}{l}\text { Grossmann } \\
\text { et al (2019) }\end{array}$ & RCT & Germany & 52 & $\begin{array}{l}\text { Standard blood analysis, } \\
\text { rheumatoid factor, anti-cyclic } \\
\text { citrullinated peptide antibodies, } \\
\text { thyroid peroxidase antibodies, } \\
\text { thyroglobulin antibodies, TSH } \\
\text { receptor antibodies, and antibodies } \\
\text { to mitochondria, adrenal cortex } \\
\text { cells and islet cells }\end{array}$ & $\begin{array}{l}\text { Hashimoto's disease, was found } \\
\text { to be the most common disease, } \\
\text { associated with premature ovarian } \\
\text { failure. Hashimoto's disease was } \\
\text { present in } 32.7 \% \text { of the cohort }\end{array}$ \\
\hline $\begin{array}{l}\text { Saglam et al } \\
(2014)\end{array}$ & RCT & Turkey & 85 & $\begin{array}{l}\text { Measurement of AMH, inhibin B, } \\
\text { gonadotrophins, estradiol, } \\
\text { testosterone, } 17 \mathrm{OH} \text { progesterone, } \\
\text { and dehydroepiandrosterone sulfate }\end{array}$ & $\begin{array}{l}\text { The serum levels of } \\
\text { gonadotrophins, sex steroids, } \\
\text { inhibin B, and free thyroid } \\
\text { hormones were similar in the two } \\
\text { groups whereas TSH was higher } \\
\text { in women with AITD. The } \\
\text { patients with AITD had } \\
\text { significantly lower levels of } \\
\text { AMH than controls }\end{array}$ \\
\hline $\begin{array}{l}\text { Samsami et } \\
\text { al }(2020)\end{array}$ & $\mathrm{RCT}$ & Iran & 98 & $\begin{array}{l}\text { TSH, thyroxine (T4), anti-TPO } \\
\text { antibody, prolactin, AMH, FSH, } \\
\text { luteinizing hormone (LH), and } \\
\text { antral follicular count (AFC) }\end{array}$ & $\begin{array}{l}\text { The patients' mean age, BMI, } \\
\text { duration of infertility, mean } \\
\text { prolactin, TSH, thyroxine and } \\
\text { FSH level did not differ } \\
\text { significantly between those two } \\
\text { groups. The results show } \\
\text { significantly lower mean AFC } \\
\text { and AMH levels in the anti-TPO } \\
\text { positive group }\end{array}$ \\
\hline $\begin{array}{l}\text { Pirgon et al } \\
(2016)\end{array}$ & RCT & Turkey & 30 & $\begin{array}{l}\text { Anti-ovarian antibody (AOAb), } \\
\text { LH/FSH ratio, estradiol, anti- } \\
\text { mullerian hormone (AMH), inhibin- } \\
\text { B, total testosterone, antral follicle } \\
\text { count, ovarian volumes and uterine } \\
\text { length }\end{array}$ & $\begin{array}{l}\text { No important differences between } \\
\text { the teenagers with HT and } \\
\text { healthy controls in relation to } \\
\text { LH/FSH ratio, estradiol and } \\
\text { inhibin-B levels, while AOAb, } \\
\text { AMH and total testosterone levels } \\
\text { were higher in HT group }\end{array}$ \\
\hline $\begin{array}{l}\text { Quintino- } \\
\text { Moro et al } \\
(\mathbf{2 0 1 4})\end{array}$ & RCT & Brazil & 66 & $\begin{array}{l}\text { Characteristics of their disease, the } \\
\text { date of their diagnosis, the time } \\
\text { since diagnosis, the results of } \\
\text { laboratory and imaging tests, to } \\
\text { confirm whether any associated } \\
\text { autoimmune diseases had been } \\
\text { diagnosed }\end{array}$ & $\begin{array}{l}\text { In the } 66 \text { women with HT } \\
\text { enrolled in that study, the } \\
\text { prevalence of infertility was } \\
47.0 \%\end{array}$ \\
\hline
\end{tabular}

\section{DISCUSSION}

The first study revealed that autoimmune thyroid diseases and especially Hashimoto's disease, is the most common disease, associated with premature ovarian failure. Hashimoto's disease was present in $32.7 \%$ of the cohort, although in general population only $6 \%$ of women below the age of 40, are affected. So, they recommend that women with premature ovarian failure should be screened for autoimmune diseases and especially those who wish to conceive. This screening can be performed by either a rheumatologist or by a gynecologist and should include a full medical history, a physical examination and screening for thyroid peroxidase. ${ }^{12}$

The second study showed that women with autoimmune thyroid disease (AITD) have a lower number of pregnancies and live births and lower AMH levels. This means that those women have a diminished ovarian reserve. AMH was proposed to be the best single marker 
of the ovarian response to ovulation induction treatment. ${ }^{17}$ Therefore, that study supports the hypothesis that women with AITD have prematurely aging ovaries. ${ }^{13}$

Undoubtedly, the third study revealed that anti-TPO positive women had AMH and AFC levels significantly lower than the anti-TPO negative women. Regarding the association between the AMH level and age, there was no significant difference between the groups of women younger than 35 years, but in women of 35 or older, levels of this hormone were significantly lower in the anti-TPO positive group. However, AFC - another criterion for ovarian reserve - was significantly lower in anti-TPO positive women in both age groups. Also, the other predictor of ovarian reserve, $\mathrm{FSH}$, was higher in the antiTPO positive group, but the difference between two groups was not significant. ${ }^{14}$ On the other hand, the fourth study demonstrated, that AMH levels were not affected by HT in adolescent girls. Actually, that study confirmed that girls diagnosed with autoimmune thyroiditis have normal ovarian reserve based on measurements of $\mathrm{AMH}$, inhibin $\mathrm{B}, \mathrm{FSH}, \mathrm{LH} / \mathrm{FSH}$ ratio, estradiol and antral follicle counts. That may happen since the autoimmune damage to the ovaries should take longer to become detectable and the teenage years might be too early to see these effects. ${ }^{15} \mathrm{On}$ the whole, we comprehend that young women with HT may not have significant differences in AMH levels and in ovarian reserve, compared to healthy women. Still, in older women with HT, those levels seem to be significantly lower. Therefore, it is important to individualize stimulation protocols according to age, ovarian reserve and endocrine status. ${ }^{17}$

The latter study estimated that the prevalence of infertility in women with HT was $47.0 \%$. Also, it was found that age $\leq 35$ years at diagnosis, had an effect on the mean number of pregnancies in this group, when half of the sample were over 35 years of age at the time of diagnosis. Furthermore, after the diagnosis of HT, pregnancies were always fewer irrespective of the woman's age. ${ }^{16}$

Thyroid autoimmunity (TAI) and/or thyroid dysfunction are prevalent in women of reproductive age and have independently been associated with adverse fertility and pregnancy outcomes, in the case of spontaneous conception or after assisted reproductive technology (ART). Despite the recent update of the guidelines by the American thyroid association (ATA) for the diagnosis and management of thyroid disease during pregnancy and the postpartum, many questions remain unsettled in ART. ${ }^{18}$

\section{CONCLUSION}

In conclusion, women with autoimmune thyroiditis might be at risk of ovarian insufficiency because of the decrease in ovarian reserve. Patients with positive anti-TPO antibody findings who have not yet completed their family, should be aware of their lower reproductive life span, before seeking medical advice for infertility. It seems important for both candidate couples and health practitioners to predict the impact of thyroid autoimmunity (TAI) on fertility outcome, after a given number of IVF/ICSI treatment cycles.

The interaction between thyroid disease and fertility is complex. Overt thyroid dysfunction frequently leads to menstrual disturbances, fertility problems and pregnancy complications and should therefore be treated accordingly.

This review endorses further randomized controlled trials to clarify the impact of Hashimoto's disease on female fertility. Thyroid evaluation should be performed in any woman who wishes to get pregnant with family history of thyroid problem or irregular menstrual cycle or more than two miscarriages or inability to conceive after 1 year of regular unprotected intercourse. It may be recommended that all patients seeking medical assistance for infertility, should be screened for underlying thyroid disease, given the potential detrimental effect of thyroid dysfunction on fertility outcome. A thorough medical history and physical examination should be the first step. Furthermore, assessment of ovarian reserve in women undergoing assisted reproduction is valuable in optimizing the treatment protocol and in counselling these patients. Thus, availability of a reliable measure of ovarian reserve is crucial for this purpose.

\section{Funding: No funding sources \\ Conflict of interest: None declared \\ Ethical approval: Not required}

\section{REFERENCES}

1. Verma I, Sood R, Juneja S, Kaur S. Prevalence of hypothyroidism in infertile women and evaluation of response of treatment for hypothyroidism on infertility. Int J Appl Basic Med Res. 2012;2(1):17-9

2. Ihnatowicz P, Drywień M, Wątor P, Wojsiat J. The importance of nutritional factors and dietary management of Hashimoto's thyroiditis. Ann Agric Environ Med. 2020;27(2):184-93.

3. Rouland A, Buffier P, Petit JM, Vergès B, Bouillet B. Thyroiditis: What's new in 2019. La Revue de medicine interne. 2020;41(6):390-5.

4. Vander Borght M, Wyns C. Fertility and infertility: Definition and epidemiology. Clin Biochem. 2018;62:2-10.

5. McDermott MT. Thyroid disease and reproductive health. Thyroid. 2004;14(1).

6. De Vivo A, Mancuso A, Giacobbe A, Moleti M, Maggio Savasta L, De Dominici R, Priolo AM, Vermiglio F. Thyroid function in women found to have early pregnancy loss. Thyroid. 2010;20(6):6337.

7. Seungdamrong A, Steiner AZ, Gracia CR, Legro RS, Diamond MP, Coutifaris C, et al. Preconceptional antithyroid peroxidase antibodies, but not thyroidstimulating hormone, are associated with decreased live birth rates in infertile women. Fertility and Sterility 2017;108(5):843-50. 
8. ACOG Practice Bulletin, Number 223. Thyroid Disease in Pregnancy. Obstetrics \& Gynecology. 2020;135(6):261-74.

9. Moher D, Liberati A, Tetzlaff J, Altman DG. Preferred reporting items for systematic reviews and meta-analyses: the PRISMA Statement. BMJ. 2009;339:b2535.

10. Modesti PA, Reboldi G, Cappuccio FP. Panethnic differences in blood pressure in Europe: a systematic review and meta-analysis. PLoS One. 2016;11(1): e0147601.

11. Herzog R, Álvarez-Pasquin MJ, Díaz C, Del Barrio JL, Estrada JM, Gil Á. Are healthcare workers' intentions to vaccinate related to their knowledge, beliefs and attitudes? a systematic review. BMC Public Health. 2013;13(1).

12. Grossmann B, Saur S, Rall K, Recher AC, Hubner S, Henes J, et al. Prevalence of autoimmune disease in women with premature ovarian failure. Eur J Contracept Reprod Health Care. 2019;25(1):72-5.

13. Saglam F, Onal ED, Ersoy R, Koca C, Ergin M, Erel O, Cakir B. Anti-Müllerian hormone as a marker of premature ovarian aging in autoimmune thyroid disease. Gynecological Endocrinology. 2014;31(2):165-8.

14. Samsami A, Ghasmpour L, Moradi Alamdarloo S, Davoodi S, Rahmati J, Karimian A, Tavasoli M. Women with Autoimmune Thyroiditis have Lower
Reproductive Life Span or Not? A Cross- Sectional Study. Int $\mathrm{J}$ Comm Based Nurs Midwifery. 2020;8(4):305-10.

15. Pirgon O, Sivrice C, Demirtas H, Dundar B. Assessment of ovarian reserve in euthyroid adolescents with Hashimoto thyroiditis. Gynecological Endocrinology. 2016;32(4):306-10.

16. Quintino-Moro A, Zantut-Wittmann DE, Tambascia M, Machado Hda C, Fernandes A. High Prevalence of Infertility among Women with Graves' Disease and Hashimoto's Thyroiditis. Int J Endocrinol. 2014:982705.

17. Muttukrishna S, McGarrigle H, Wakim R. Antral follicle count, anti-Mullerian hormone and inhibin B: predictors of ovarian response in assisted reproductive technology? BJOG. 2005;112:1384-90.

18. Unuane D, Velkeniers B. Impact of thyroid disease on fertility and assisted conception. Best Pract Res Clin Endocrinol Metabol. 2020;34(4):101378.

Cite this article as: Sarantaki A, Tsiorou K, Gourounti K. The impact of Hashimoto's disease on female fertility: a systematic review. Int J Reprod Contracept Obstet Gynecol 2022;11:583-9. 